\title{
Routes of clearance of radioactive water from the rabbit vitreous
}

\author{
H. MOSELEY, ${ }^{1}$ W. S. FOULDS,${ }^{2}$ D. ALLAN,${ }^{1}$ AND P. M. KYLE ${ }^{2}$ \\ From the ${ }^{1}$ West of Scotland Health Boards, Department of Clinical Physics and Bio-Engineering, \\ 11 West Graham Street, Glasgow G49LF, and the ${ }^{2}$ Tennent Institute of Ophthalmology, University of Glasgow, \\ Western Infirmary, Glasgow G11 6NT
}

SUMMARY The movement of water to and from the vitreous across the retina and pigment epithelium is important in relation to an understanding of such conditions as retinal detachment and its surgical cure, central serous retinopathy, and retinal oedema. Experiments were carried out to determine the main routes for removal of water injected into the vitreous and to see if the removal could be explained on the basis of diffusion or whether bulk flow was also implicated. $25 \mu \mathrm{Ci}$ of ${ }^{3} \mathrm{H}_{2} \mathrm{O}$ in $25 \mu$ l were injected into a central position in the vitreous humour of living rabbits under general anaesthesia. For 9 animals blood was collected from one of the 4 vortex veins draining the choroid and the radioactivity in the samples measured. For another 6 rabbits similarly injected the radioactivity in samples of aqueous humour was determined. The percentage of injectate recovered from the vortex vein blood ranged between $13 \%$ and $38 \%$, mean $25 \pm 3 \%$. The percentage of injectate recovered from the aqueous humour ranged between $1 \cdot 2 \%$ and $5 \cdot 2 \%$, mean $2 \cdot 8 \pm 0 \cdot 6 \%$. Analysis of the time course of isotope activity in the samples revealed a mean transit time from the mid vitreous to the choroid of $32 \pm 2$ minutes, and from the mid vitreous to the anterior chamber of $84 \pm 3$ minutes. By means of a computer model it was calculated that diffusion alone could effect this transfer; if active transport were involved in the transport of ${ }^{3} \mathrm{H}_{2} \mathrm{O}$ to the choroid, this was not a limiting factor under the conditions of the experiment.

The movement of water to and from the vitreous across the retina and retinal pigment epithelium (RPE) is important in relation to the understanding of such problems as retinal detachment and its surgical cure, central serous retinopathy, and retinal oedema. The exchange of fluid posteriorly to the choriocapillaris might be affected by the barrier function of the RPE or by active transport through the cells of the RPE. Absorption of subretinal fluid after successful retinal detachment surgery has been suggested as evidence for the existence of a bulk flow of fluid across the RPE to the choroid, but attempts to measure a pressure difference across the RPE have been unsuccessful. ${ }^{1}$

Water movement into and out of the vitreous has received little attention in the literature. In 1942 Kinsey et al. ${ }^{2}$ studied movement of water from the blood into the vitreous in 2 rabbits using heavy water and gravimetric techniques for its detection. All other Correspondence to Dr H. Moseley. reported work on molecular movement from the vitreous has used a variety of tracers, some of which might have been subject to the processes of active transport, or differed from water in intermolecular interactions or in molecular size.

Diffusional exchange with the water in the anterior chamber, which is largely cleared by bulk flow through the outflow apparatus, is another factor which might influence the clearance of water from the vitreous.

As part of a study of water movement in the eye, experiments were carried out to: (1) determine the main routes of clearance of ${ }^{3} \mathrm{H}_{2} \mathrm{O}$ injected into the midvitreous of living rabbit eyes and to quantify the movement via each of these routes; (2) determine by comparison with a mathematical model whether any detected movements of water could be explained entirely by diffusion or whether active transport or bulk flow were involved. The results of these studies are presented in this paper. 


\section{Materials and methods}

CLEARANCE TO THE CHOROID

Experiments were performed on nine male Dutch rabbits weighing between 1.6 and $2.5 \mathrm{~kg}$. Details of the surgical technique are described elsewhere. ${ }^{3} 25$ $\mu \mathrm{Ci}$ of ${ }^{3} \mathrm{H}_{2} \mathrm{O}$ in $25 \mu$ l water was injected into a midvitreal position via the pars plana ciliaris, and blood was collected in a series of long-form Pasteur capillary pipettes from the superior nasal vortex vein, which had previously been dissected free of episcleral tissue and divided transversely. The narrow end of each pipette was placed against the open end of the vein, and the pipette was inclined downwards away from the eye at a slight angle. As the blood left the vein it was taken into the pipette by capillary attraction and then travelled down the pipette by gravity assisted capillary action. After 2 minutes the pipette was removed and a new one placed in position. The contents of each pipette were blown into a preweighed scintillation vial. After this the pipette still contained some blood, and so it was then washed through with 1 $\mathrm{ml}$ of water into another scintillation vial. On some occasions the pipette was filled with blood before the 2-minute collection period was completed, and in these cases the pipette changes were carried out more frequently. There was no significant blood loss during the procedure, virtually all of the blood leaving the vortex vein being collected in the pipettes.

The scintillation vials containing the blood samples were weighed again after the experiment and the volume of blood calculated. The vials were kept capped throughout to prevent contamination and evaporative losses.

The amount of blood in each vial was approximately $0.2 \mathrm{ml}$. Without further sample preparation the counting efficiency in the liquid scintillator would have been low because of the quenching effect of the blood. To overcome this the wet oxidation procedure described by Mahin and Lofberg ${ }^{4}$ was employed. On completion of the solubilisation process $10 \mathrm{ml}$ of a multipurpose xylene-based liquid scintillator NE260 (Nuclear Enterprises Ltd) were added to the vial. Samples were counted in a liquid scintillation spectrometer, and a standard channels ratio quench correction procedure was carried out. ${ }^{5}$ After each experiment was completed the eye was enucleated and the number of vortex veins counted.

\section{CLEARANCE VIA THE ANTERIOR CHAMBER}

Other experiments were performed on a further 6 male Dutch rabbits of 1.7 to $2.3 \mathrm{~kg}$ weight. The experimental method was as described above except that, after the injection of the $25 \mu \mathrm{Ci}$ of ${ }^{3} \mathrm{H}_{2} \mathrm{O}$ into the mid vitreous, samples of aqueous humour were removed from the anterior chamber by cannulation.
Table 1 Results of experiments in which ${ }^{3} \mathrm{H}_{2} \mathrm{O}$ was detected in the blood of a vortex vein after the isotope had been injected into the vitreous

\begin{tabular}{lll}
\hline $\begin{array}{l}\text { Experiment } \\
\text { number }\end{array}$ & $\begin{array}{l}\text { Amount of }{ }^{3} \mathrm{H}_{2} \mathrm{O} \\
\text { recovered from } \\
\text { vortex vein (\%) }\end{array}$ & $\begin{array}{l}\text { Mean } \\
\text { transit } \\
\text { time }(\mathrm{min})\end{array}$ \\
\hline 1 & 18 & 36 \\
2 & 38 & 24 \\
3 & 23 & 36 \\
4 & 35 & 27 \\
5 & 27 & 32 \\
6 & 24 & 39 \\
7 & 26 & 30 \\
8 & 24 & 26 \\
9 & 13 & 34 \\
Mean & 25 & 32 \\
Standard deviation & 8 & 5 \\
Standard error & 3 & 2 \\
\hline
\end{tabular}

Samples of $0.2 \mathrm{ml}$ of aqueous were taken at intervals of 15 minutes for 4 hours. The deficiency of aqueous was made good immediately by replacement with isotonic saline.

Again the radioactivity in the samples was assayed by liquid scintillation counting with quench correction, but, unlike the blood samples, no extra sample treatment was needed in this case.

\section{Results}

CLEARANCE TO THE CHOROID

All the experiments lasted 80 minutes. The summation of the counts from the samples in each experiment was determined and, by comparison with standards the percentage of injected ${ }^{3} \mathrm{H}_{2} \mathrm{O}$ collected was obtained (Table 1). It ranged between $13 \%$ and

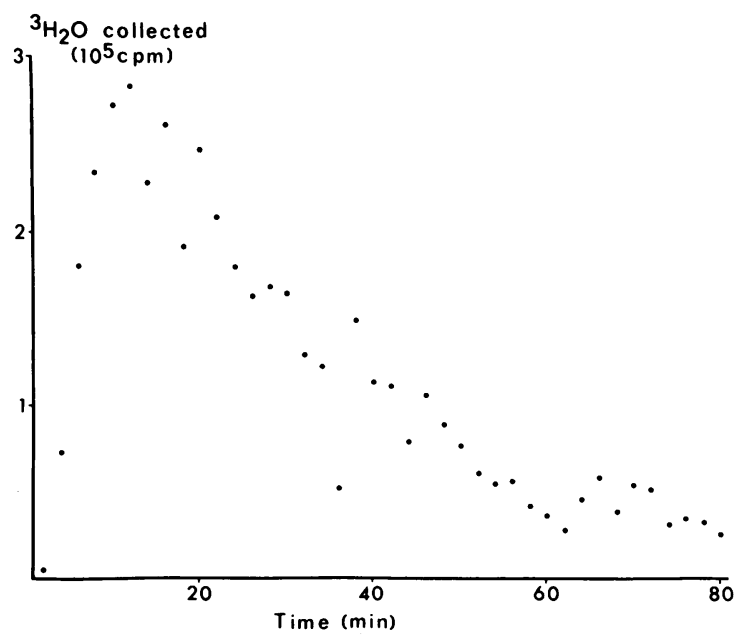

Fig. 1 Typical result showing the appearance of ${ }^{3} \mathrm{H}_{2} \mathrm{O}$ in the blood of a vortex vein following its injection into the vitreous. 


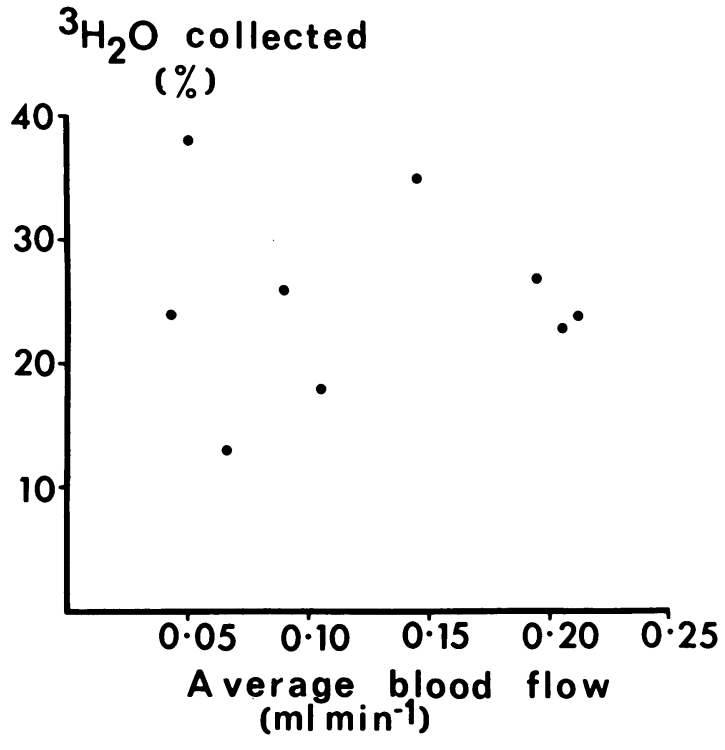

Fig. 2 Percentage of ${ }^{3} \mathrm{H}_{2} \mathrm{O}$ collected from a vortex vein plotted against average blood flow in the vortex vein during the course of the experiment.

$38 \%$, with a mean of $25 \%$ and a standard error of $3 \%$.

The results from a typical experiment showing the appearance of isotope in the vortex vein at different times after intravitreal injection are given in Fig. 1. For each experiment the mean transit time for the movement of ${ }^{3} \mathrm{H}_{2} \mathrm{O}$ from a central position in the vitreous to the choroidal vasculature was calculated. The mean transit time is the average time for the transfer of tracer from injection site to collection point and is given in Table 1 . It varied from 26 minutes to 39 minutes, with a mean of 32 minutes and standard error of 2 minutes.

It is relevant to consider whether the amount collected bore any relation to the vortex vein blood

Table 2 Results of experiments in which ${ }^{3} \mathrm{H}_{2} \mathrm{O}$ was detected in samples drawn from the anterior chamber after the isotope had been injected into the vitreous

\begin{tabular}{lll}
\hline $\begin{array}{l}\text { Experiment } \\
\text { number }\end{array}$ & $\begin{array}{l}\text { Amount of }{ }^{3} \mathrm{H}_{2} \mathrm{O} \\
\text { recovered from the } \\
\text { anterior chamber (\%) }\end{array}$ & $\begin{array}{l}\text { Mean } \\
\text { transit } \\
\text { time (min) }\end{array}$ \\
\hline 1 & $1 \cdot 2$ & 90 \\
2 & $5 \cdot 2$ & 72 \\
3 & $2 \cdot 0$ & 93 \\
4 & $3 \cdot 0$ & 86 \\
5 & $1 \cdot 6$ & 78 \\
6 & $3 \cdot 5$ & 83 \\
Mean & $2 \cdot 8$ & 84 \\
Standard deviation & $1 \cdot 5$ & 8 \\
Standard error & 0.6 & 3 \\
\hline
\end{tabular}

flow. This is plotted in Fig. 2, where it can be seen that there was no correlation ( $p>0.5)$ between these parameters. Thus the amount of ${ }^{3} \mathrm{H}_{2} \mathrm{O}$ collected in a single vortex vein was not dependent on the rate of blood flow.

Since the mean transit time is the average time taken for the tracer to reach the vortex vein in each experiment, it was of interest to see if any relation existed between this and the percentage of ${ }^{3} \mathrm{H}_{2} \mathrm{O}$ collected from the vortex vein. This is plotted in Fig. 3 , where it can be seen that the 2 variables are clearly related $(0.02<\mathrm{p}<0.05)$.

On each animal it was confirmed that 4 vortex veins were present in the rabbit eye under investigation.

\section{CLEARANCE VIA THE ANTERIOR CHAMBER}

All the experiments lasted 4 hours. Again the summation of the counts from samples in each experiment was determined and compared with standards to determine the percentage of injected ${ }^{3} \mathrm{H}_{2} \mathrm{O}$ collected in the aqueous samples. The results are shown in Table 2 and vary from $1.2 \%$ to $5.2 \%$, with a mean of $2.8 \%$ and a standard error of $0.6 \%$. Results for a typical experiment showing the appearance of isotope in the aqueous at different times after intravitreal injection are given in Fig. 4. For each experiment the mean transit time for the movement of ${ }^{3} \mathrm{H}_{2} \mathrm{O}$ from a

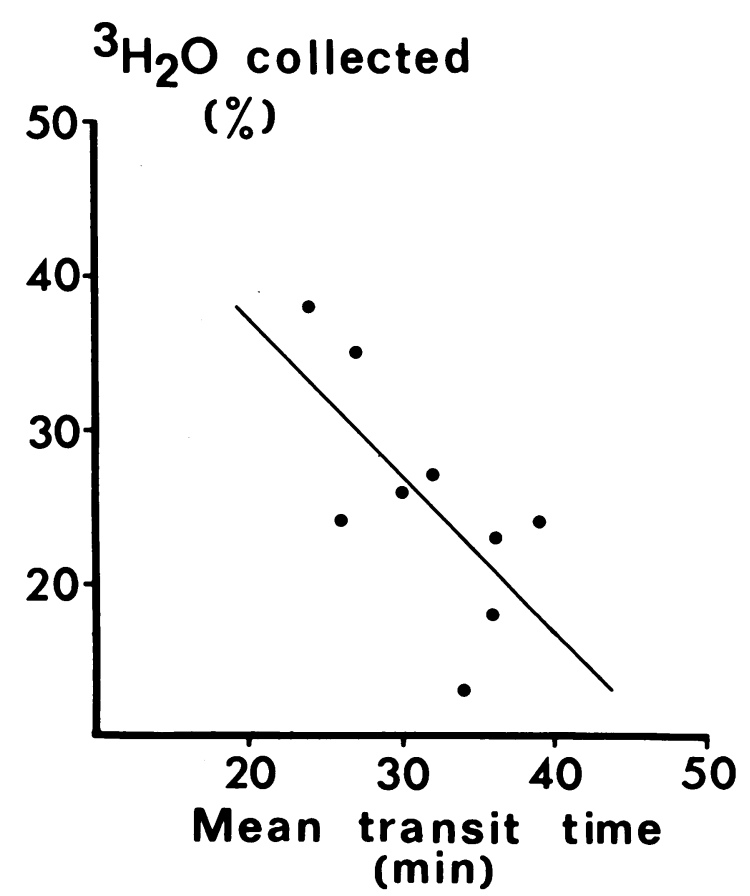

Fig. 3 Percentage of ${ }^{3} \mathrm{H}_{2} \mathrm{O}$ collected from a vortex vein plotted against mean transit time. 


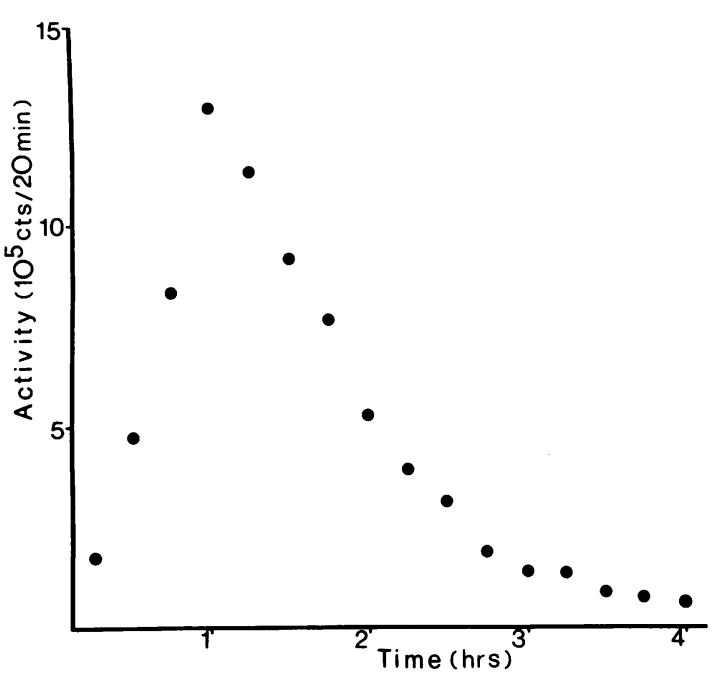

Fig. 4 Typical result showing the appearance of ${ }^{3} \mathrm{H}_{2} \mathrm{O}$ in the samples of aqueous humour following its injection into the vitreous.

central position in the vitreous to the anterior chamber was calculated, and these results are also shown in Table 2. Mean transit times varied from 72 minutes to 93 minutes, with an average of 84 minutes and a standard error of 3 minutes.

\section{Discussion}

If it is assumed that each vortex vein drains one quarter of the choroid, as verified experimentally in the rabbit, ${ }^{6}$ the total amount of tritiated water removed by the choroid averaged $100 \%$ with a standard error of $12 \%$. This average figure of $100 \%$ is fortuitous for undoubtedly not all of the injectate is cleared via the choroid. Taking into consideration the standard error of $12 \%$ in the mean recovery, it would appear that somewhat in excess of $88 \%$ of the injected ${ }^{3} \mathrm{H}_{2} \mathrm{O}$ was cleared from the mid vitreous to the choroid and a much smaller proportion $(2 \cdot 8 \% \pm 0 \cdot 6 \%)$ cleared by way of the anterior chamber.

The figure for the anterior chamber route can be regarded as only an approximate figure at this stage. It is clear, for example, that during the 15 minutes between samples some ${ }^{3} \mathrm{H}_{2} \mathrm{O}$ must be removed by normal aqueous outflow processes, diffusion into the blood in iris vessels, and diffusion through the cornea, but calculations suggest that this should be only a small fraction of the total ${ }^{3} \mathrm{H}_{2} \mathrm{O}$ detected in the aqueous samples. ${ }^{7}$

Some experiments to detect the ${ }^{3} \mathrm{H}_{2} \mathrm{O}$ appearing on the outer surface of the sclera have also been performed. ${ }^{7}$ The measurements indicate that only 1 or $2 \%$ of the ${ }^{3} \mathrm{H}_{2} \mathrm{O}$ leaves the eye by this route. This small amount is consistent with the results presented here and parallels the results of earlier studies with radioactive xenon tracer. ${ }^{8}$

In the rabbit with an anangiotic retina no clearance by way of the retinal circulation was to be expected, but this route might well be important in species with a holangiotic retina such as man.

The mean transit time for a molecule of intravitreally injected ${ }^{3} \mathrm{H}_{2} \mathrm{O}$ to reach the choroid was $32 \pm 2$ minutes and to reach the anterior chamber $84 \pm 3$ minutes.

Bill 6 has shown that in the rabbit each of the 4 vortex veins drains one quarter of the blood in the choroid. In our experimental eyes each was demonstrated as possessing 4 vortex veins. Although a mean recovery of injectate of $25 \% \pm 3 \%$ was obtained from the one vortex vein sampled from each eye, suggesting that virtually all the injectate was cleared via the choroid, there was considerable scatter of results between experiments.

Various possible causes of the variations in recovery figures might be considered. These include inherent radioactivity counting errors, variations in blood flow induced by the sectioning of a vortex vein, clearance of ${ }^{3} \mathrm{H}_{2} \mathrm{O}$ by way of the anterior chamber, and variations in the initial position of the injectate relative to the centre of the vitreous.

The random nature of the radioactive disintegration process is characterised by the Poisson probability distribution, for which the standard deviation is equal to the square root of the counts recorded, and this gives a measure of the counting error. In the case of the present experiments this was less than $0 \cdot 1 \%$.

Sectioning of a vortex vein might induce a preferential increase in flow in that vein. However, it is likely that the effect would be similar in each animal and so would not contribute significantly to the spread of recovery results.

The amount of ${ }^{3} \mathrm{H}_{2} \mathrm{O}$ cleared by way of the anterior chamber is too small to account for the magnitude of recovery variations found from the choroidal blood samples.

Differences in the positioning of the injection needle seem the most likely cause of the recovery variations. If the injectate was initially closer to choroid drained by an intact vortex vein than to the area drained by the sectioned vein, then a higher proportion of the ${ }^{3} \mathrm{H}_{2} \mathrm{O}$ is likely to have been cleared by veins other than that being sampled. The measured recovery would then be lower than for an experiment with a more centrally placed tip. The spread of recovery figures about the mean is thus probably due to variations in the solid angle subtended within the eye by the region of the choriocapillaris drained by the vortex vein being sampled.

Interexperimental differences in the initial position 
of the injectate are also likely to explain the relation between the mean transit time for clearance to the choroid and the percentage of ${ }^{3} \mathrm{H}_{2} \mathrm{O}$ recovered, since the positioning needed to cause a high recovery fraction lead to a shorter mean transit time for the water molecules. Variations in the initial position of injectate probably contribute, along with other factors, to the range of mean transit times recorded.

Thus the correlation between the amount of isotope collected from a vortex vein and mean transit time can be explained by the tracer's being injected at different distances from the vortex vein's 'catchment area.' This presupposes a direct line of communication between the vitreous and the choriocapillaris. The correlation would not hold if the isotope entered the vortex vein via the anterior chamber, and in any case the measurements of ${ }^{3} \mathrm{H}_{2} \mathrm{O}$ recovered from the aqueous show this route to be unimportant. The belief that the tracer entered the vortex vein from the choroidal vasculature is further supported by the agreement between the experimental results and the model outlined below, in which the isotope could leave the eye only from within the choroid.

In an earlier paper $^{3}$ it was shown that the mean transit time for xenon to the choroid was 28 minutes, and this is very similar to the mean transit time for tritiated water in the present experiments (32 minutes), in spite of the fact that the diffusion coefficient for tritiated water is almost twice that of xenon. It is, however, difficult to predict the effect of the diffusion coefficient on the average time for tracer molecules to appear in the choroidal blood following their introduction into the vitreous humour. The geometry is complex, and tracer molecules are moving to the front as well as to the back of the eye. By substituting different diffusion coefficients in the mathematical model described below, it turns out that altering the diffusion coefficient by $80 \%$ changes the mean transit time to the choroid by only $15 \%$, which is in reasonable agreement with the experimental values found for xenon and tritiated water.

From experiments in which heavy water was injected intraperitonealy and subsequently detected in the vitreous of 2 rabbits Kinsey et al. ${ }^{2}$ calculated that one-half of the vitreous volume was replaced every 10 to 15 minutes, i.e., 75 to $87.5 \%$ is replaced in 30 minutes. The present study shows that the mean transit time of water from the mid vitreous to a vortex vein is $32 \pm 2$ minutes, and this finding is in good agreement with the earlier work of Kinsey et al. ${ }^{2}$

A difficulty in the interpretation of the results might arise if a significant amount of ${ }^{3} \mathrm{H}_{2} \mathrm{O}$ passed into the general blood circulation for a time before collection. To explore this question samples of blood were taken from the ear vein of a rabbit at various times during one experiment. These samples were analysed in the same way as those from the vortex vein. The amount of ${ }^{3} \mathrm{H}_{2} \mathrm{O}$ activity in the ear vein samples showed that no more than $1 \%$ of the ${ }^{3} \mathrm{H}_{2} \mathrm{O}$ collected from the vortex vein could be attributed to ${ }^{3} \mathrm{H}_{2} \mathrm{O}$ in the general circulation. Such a small fraction does not invalidate the proposed interpretation.

The experiments described show quite clearly that removal of tritiated water injected into the mid vitreous of the rabbit is largely by way of the choroid and only to a small extent by other routes such as the anterior chamber.

The movement to the choroid and anterior chamber having been quantified and its rate determined there still remained the need to determine whether the tracer movement observed could be explained entirely by diffusional movement.

In order to study this a mathematical model was developed in which tracer in the eye was allowed to diffuse from the vitreous into the surrounding tissue with removal occurring in the choriocapillaris. The model was initially applied to the diffusion of xenon ${ }^{3}$ in order to reduce the effect of complex interactions between the tracer and the surrounding tissues. The results obtained with the model were in good agreement with those obtained experimentally. The validity of the model for the diffusion of xenon having been established, it may now be applied to water.

The diffusion coefficients for ${ }^{3} \mathrm{H}_{2} \mathrm{O}$ in the various ocular tissues were taken to be the published values for xenon ${ }^{9}$ multiplied by the ratio of the self-diffusion coefficient of water to that of xenon in water. If the self-diffusion coefficient of water as measured by Wang ${ }^{10}$ and the diffusion coefficient of xenon in water as measured by Unsworth and Gillespie ${ }^{11}$ are taken, this ratio has a value of 1.8 between $20^{\circ} \mathrm{C}$ and $37^{\circ} \mathrm{C}$. The diffusion coefficients of ${ }^{3} \mathrm{H}_{2} \mathrm{O}$ were thus calculated to be $2.34 \times 10^{-5} \mathrm{~cm}^{2} \mathrm{sec}^{-1}$ in sclera, $1.13 \times 10^{-5} \mathrm{~cm}^{2} \mathrm{sec}^{-1}$ in combined retina and choroid, and $1.73 \times 10^{-5} \mathrm{~cm}^{2} \mathrm{sec}^{-1}$ in vitreous. The model is described in detail elsewhere. ${ }^{712}$

The removal of ${ }^{3} \mathrm{H}_{2} \mathrm{O}$ by the choroidal blood according to the model is shown in Fig. 5. The curves predicted by the model and those obtained experimentally are in reasonable agreement. The mean transit time was calculated from the rate of appearance of ${ }^{3} \mathrm{H}_{2} \mathrm{O}$ in the choroidal blood according to the model for a time which was equal to the duration of the experiments. The forecast from the model of a mean transit time in the range 28 to 36 minutes, when the needle is within $0.5 \mathrm{~mm}$ of the centre of the eye is in good agreement with the experimental value of $32 \pm 2$ minutes. It has been previously shown ${ }^{3}$ that the range in mean transit times observed experimentally corresponded to the spread in values predicted by the model for small changes in 


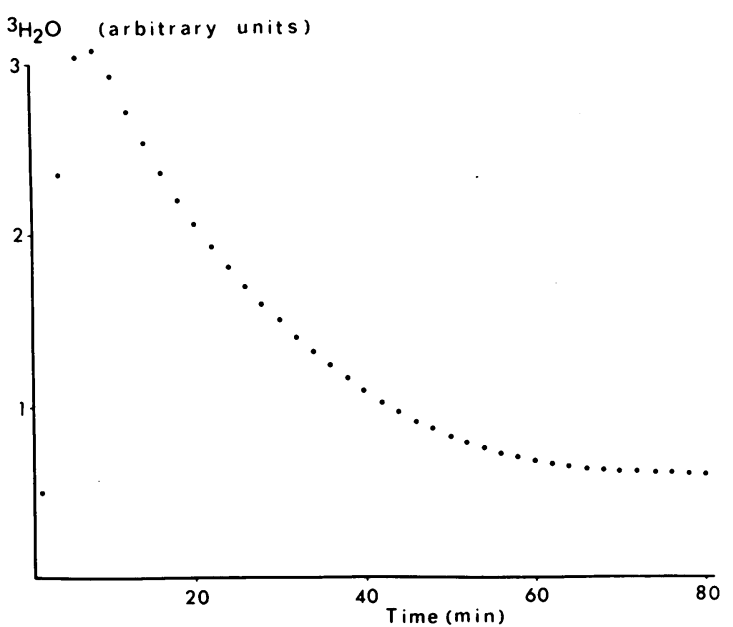

Fig. 5 Relative concentration of ${ }^{3} \mathrm{H}_{2} \mathrm{O}$ in the choroidal blood according to the model for diffusion of a bolus of $25 \mu \mathrm{l}$ in the vitreous.

the injection site. Moreover, the lack of correlation between mean transit time and rate of choroidal blood flow was a feature of both experiment and model. It is considered, therefore, that the model has provided a satisfactory explanation for the experimental results obtained with ${ }^{3} \mathrm{H}_{2} \mathrm{O}$.

In one respect the results for the model differed from the experimental data. In the model the concentration of tritiated water in the choroidal blood levels off some 60 minutes after intravitreal injection, whereas in the experimental condition (Fig. 1) the concentration continued to fall after this time. As the mathematical model allows for removal only within the choroid, and as we have shown that some of the injectate is removed via the anterior chamber, it would appear that the model is not applicable beyond 60 minutes, when presumably other exit routes begin to become more important. The mathematical model is not an exact reproduction of the eye, but it does explain the early massive diffusion of tracer to the choroid. It does not account for any slow movement of small amounts of isotope by other routes, or indeed a possible slow bulk movement of fluid to the choroid. The model is at best a mathematically idealised approximation.

The experimental results and those obtained from the mathematical model are consistent with a movement of water from the vitreous by diffusion, subsequent removal occurring principally by way of the blood flowing in the choroid and only marginally by way of the anterior chamber.

The consistency of the results with the diffusional model does not prove that there were no other processes involved in the transfer of water. Some evidence has been presented in the past for the existence of active transport of certain substances, such as organic anions, from the vitreous in vivo. ${ }^{1314}$ Moreover, in-vitro experiments on the retinal pigment epithelium of frogs and toads have shown the likelihood of an active transport system within this layer. ${ }^{15-18}$ If in our experiments the water molecules were actively transported at any stage, active transport was not a limiting factor under the conditions of the experiment.

It is believed that the present experiments have shown that: (1) There is a rapid turnover of water molecules in the vitreous. (2) Most of the vitreal water is removed by way of the choroidal vasculature, with only a small amount being cleared via the anterior chamber or sclera. The mean transit time from mid vitreous to choroid is $32 \pm 2$ minutes and from mid vitreous to anterior chamber, $84 \pm 3$ minutes. (3) The transfer of water to the choroid and anterior chamber is consistent with an explanation based on the movement of water by diffusion but does not rule out in addition active transport or a slow bulk flow.

\section{References}

1 Maurice DM, Salmon J, Zauberman H. Subretinal pressure and retinal adhesion. Exp Eye Res 1971; 12: 212-7.

2 Kinsey VE, Grant M, Cogan DG. Water movement and the eye. Arch Ophthalmol 1942; 27: 242-52.

3 Moseley H, Foulds WS. The movement of xenon-133 from the vitreous to the choroid. Exp Eye Res 1982; 34: 169-79.

4 Mahin DT, Lofberg RT. A simplified method of sample preparation for determination of tritium, carbon-14 or sulfur-35 in blood or tissue by liquid scintillation counting. Anal Biochem 1966; 16: 500-9.

5 Belcher EH. Measurements of radioactivity in vitro. In: Belcher $\mathrm{EH}$, Vetter H, eds. Radioisotopes in medical diagnosis. London: Butterworths, 1971: 79-117.

6 Bill A. Quantitative determination of uveal blood flow in rabbits. Acta Physiol Scand 1962; 55: 101-10.

7 Moseley H. Studies of fluid movement in the eye. $\mathrm{PhD}$ thesis. University of Glasgow, 1980.

8 Moseley H, Johnson NF, Foulds WS. Vitreo-scleral fluid transfer in the rabbit. Acta Ophthalmol (Kbh) 1978; 56: 769-76.

9 Strang R. The determination of the diffusion coefficient of krypton in rabbit ocular tissue. Invest Ophthalmol Visual Sci 1977; 16: 83-6.

10 Wang JH. Self-diffusion coefficients of water. J Phys Chem 1965; 69: 4412 .

11 Unsworth J, Gillespie FC. Diffusion coefficients of xenon and krypton in water from $0^{\circ} \mathrm{C}$ to $80^{\circ} \mathrm{C}$ and in biological tissues at $37^{\circ} \mathrm{C}$. In: Sherwood JN, Chadwick AV, Muir WM, et al., eds. Proceedings of the Thomas Graham memorial symposium. London: Gordon and Breech, 1970.

12 Moseley $\mathrm{H}$. Mathematical model of diffusion in the vitreous humour of the eye. Clin Phys Physiol Meas 1981; 2: 175-82.

13 Forbes M, Becker B. The transport of organic anions by the rabbit eye. II, in vivo transport of isodopyracet (diodrast). Am J Ophthalmol 1960; 50: 867-75.

14 Cunha-Vaz JG, Maurice DM. The active transport of fluorescein by the retinal vessels and the retina. $J$ Physiol 1967; 191: 467-86.

15 Lasansky A, De Fisch FW. Potential, current and ionic fluxes across the isolated retinal pigment epithelium and the choroid. $J$ Gen Physiol 1966; 49: 913-24. 
16 Christiansson J, Palm E. The exchange of substances in the anterior part of the vitreous body, bordering upon the lens. Acta Ophthalmol $(K b h)$ 1954; 32: 197-212.

17 Tronche P, Meyniel G, Alfieri R, Roucher F, Pages C.
Répartition et metabolisme du radiophosphore 32 injecté dans le gel vitréen. Ann Oculist (Paris) 1963; 196: 159-79.

18 Miller SS, Steinberg RH. Active transport of ions across the frog retinal pigment epithelium. Exp Eye Res 1977; 25: 235-48. 